\title{
Untersuchungen über das Blut der Ascidien.
}

I. Mitteilung.

Die Vanadiumverbindung der Blutkörperchen.

Von

M. Henze.

(Aus der chemisch-physiologischen Abteilung der Zoologischen Station in Neapel.)

(Der Redaktion zugegangen am 27. Mai 1911.)

Über das Blut der Ascidien existieren in chemischer Hinsicht so gut wie keine Angaben, und doch glaube ich schon in dieser ersten Mitteilung zeigen zu können, daß dasselbe außerordentlich viel Interessantes und von den gewohnten Verhältnissen Abweichendes bietet.

Die nachstehenden Mitteilungen betreffen einstweilen ausschließlich die im Mittelmeer ziemlich verbreitete, große Ascidienform, die als Ascidia oder Phallusia mamillata bekannt ist, und deren Mantel z. B. auch das Material zum Nachweis des Vorkommens rein pflanzlicher Cellulose im Tierreiche lieferte. ${ }^{1}$ )

Die ersten Beobachtungen über die chemischen Eigenschaften des Ascidienblutes stammen von Harless ${ }^{2}$ ) aus dem Jahre 1847. Harless behauptete, das anfangs farblose Blut der Phallusia färbe sich in wenigen Minuten an der Luft tiefblau, während es beim Durchleiten von Sauerstoff farblos bleibe. Umgekehrt könne durch wenige Blasen Kohlensäure Blaufärbung hervorgerufen werden, welcher Farbton wiederum durch die Einwirkung eines Sauerstoffstromes verschwinde. Alkohol oder Äther sollen sodann angeblich sofort wieder tiefes Blau

1) E.Winterstein, Zur Kenntnis der Tiercellulose oder des Tunicins, Diese Zeitschrift, Bd. 18, S. 43.

2) Harless, Über das blaue Blut einiger wirbelloser Tiere und dessen Kupfergehalt, Müllers Archiv, 1847, S. 148. 
erzeugen. Krukenberg ${ }^{1}$ ) bestätigte im allgemeinen diese Angaben und zwar auch für einige andere Ascidienarten, obwohl er meint, daß das durch Kohlensäure blau gewordene Blut durch Sauerstoff nicht wieder vollständig zu entfärben sei. Auf einige weitere Notizen in bezug auf das Verhalten des Blutes gegenüber Säuren und Alkalien, sowie auf die durch keine Experimente gestützten Spekulationen über die respiratorische Bedeutung, mit der obengenannte Farbenänderungen verknüpft sein sollten, ist unnötig einzugehen. Diese Farbenänderungen sind überhaupt nicht $\mathrm{zu}$ beobachten. Hierauf hat neuerdings bereits Winterstein ${ }^{2}$ ) hingewiesen, der dieselben ebenso wenig wie ich beobachten konnte. Winterstein zeigte dagegen unter Anwendung exakter Methoden, daß dem Blute (Phallusia) kein spezifisches Sauerstoffbindungsvermögen zukommt, sich also augenscheinlich keine sauerstoffbindenden Pigmente oder Eiweißkörper im Blute finden, die etwa dem Hämoglobin oder Hämocyanin an die Seite zu stellen wären. Aufgefallen war Winterstein der sehr geringe Kohlensäuregehalt (nur $0,25 \%$ ), wovon ich mich gleichfalls überzeugen konnte. Der Grund dafür wird sofort verständlich, denn wie unten gezeigt werden wird, reagiert das Blut im Gegensatz zu allen bisherigen Erfahrungen stark sauer.

Griffiths, ${ }^{3}$ ) der über das Vorkommen und die Isolierung eines «Achroglobins» mit Sauerstoff fixierenden Eigenschaften berichtet hat, wurde schon von Winterstein abgefertigt. Wie fast alle Angaben genannten Autors auf diesem Gebiete ist

1) Krukenberg, Das Chromogen in den Blutkörperchen einiger Ascidien, vgl. Studien, I. Reihe, 5. Abteilung, S. 100.

Weitere Beiträge zum Verständnis und zur Geschichte der Blutfarbstoffe bei den wirbellosen Tieren, ibid., I. Reihe, 5. Abt., S. 49.

Zur vergleichenden Physiologie der Lymphe, Hydro- und Hämolymphe, ibid., II. Reihe, 1. Abt., S. 92.

Die Pigmente, ibid., II. Reihe, 3. Abt., S. 48.

2) H. Winterstein, Zur Kenntnis der Blutgase wirbelloser Tiere, Biochem. Zeitschrift, Bd. 29, S. 384 .

3) Griffiths, Sur la $\gamma$-Achroglobine, nouvelle globine respiratoire, C. R. A. S., Bd. 115 , S. 738 . 
auch dieses Achroglobin eine Erfindung. (Vgl. z. B. die zitierte Arbeit von Winterstein.)

Eigene Beobachtungen.

Das Blut der Phallusia gewinnt man am einfachsten, indem man an der Basis des vorher sauber gereinigten Tieres den Mantel vorsichtig abträgt, wobei man auf die dort verlaufenden Blutgefäße und das Herz trifft. Letzteres wird behutsam angestochen, ohne den Verdauungstrakt zu verletzen. Dann tropft das Blut als farblose trübe Flüssigkeit aus und man gewinnt aus den größeren Tieren bis zu $40 \mathrm{ccm}$ und mehr.

Von einer Gerinnung des Blutes ist nichts zu beobachten. Dagegen ballen sich die Blutkörperchen leicht zusammen, wie eine mikroskopische Prüfung sofort zeigt. Im Laufe der Zeit färben sich diese Flocken bräunlich und nach längerem Stehen grünlichblau, insbesondere die am Rande des Glasgefäßes hängen gebliebenen Flöckchen. Außer den sehr kleinen Blutkörperchen, die bei mikroskopischer Betrachtung oft maulbeerartige Formen zeigen und übrigens auch histologisch noch wenig untersucht sind, beobachtet man noch vereinzelte gelbliche Pigmentzellen.

Was als erstes in Erstaunen setzt, ist die Reaktion des Blutes. Dasselbe reagiert ausgesprochen sauer auf Lackmus, und jeder Einschnitt, den man in den von Blutgefäßen durchzogenen Mantel des Tieres macht und mit einem Lackmuspapier betupft, färbt dasselbe deutlich rötlich. Viele andere Ascidienarten, die ich in dieser Hinsicht prüfte, zeigten das gleiche Verhalten.

Durch Zentrifugieren senken sich die Blutkörperchen. Eine vorläufige Prüfung der osmotischen Verhältnisse, d. h. Bestimmung der Gefrierpunktserniedrigung des Plasmas ergab eine völlige Isotonie desselben mit dem äußeren Milieu (Meerwasser), in dem die Tiere leben. Es wurde gefunden:

$$
\begin{aligned}
\text { Plasma: } \Delta & =-2,12 \\
\text { Seewasser: } \Delta & =-2,07 .
\end{aligned}
$$

Das Plasma ist schwach eiweißhaltig. Beim Zusatz von Alkohol und Erhitzen fallen Eiweißflocken aus. Nach einigen 
quantitativen Bestimmungen beträgt der Eiweißgehalt nicht mehr als ungefähr $0,2 \%$. Dieser koagulierte Eiweißkörper zeigt die allgemeinen Proteinreaktionen (Millon-, Biuret- und Xanthoproteinreaktion).

Einige Titrationen des vom Eiweiß befreiten Plasmas mit Phenolphthalein als Indikator ergaben, daß zur Neutralisation von $100 \mathrm{ccm}$ Serum ca. $5 \mathrm{ccm} \mathrm{n/10-NaOH}$ und meist noch mehr nötig waren.

Ganz außerordentlich sauer reagieren die Blutkörperchen. Bringt man mit der Spitze eines Glasstabes eine Spur des Blutkörperchenbreies auf ein Lackmuspapier, so hat man die Erscheinung, als habe man einen Tropfen starke Mineralsäure auffallen lassen. Folgende Angabe mag eine ungefähre Vorstellung von dem Säuregehalt geben. Verrührt man die abzentrifugierten Blutkörperchen mit destilliertem Wasser und bringt sie dadurch zur Hydrolysation, so benötigt die abzentrifugierte Flüssigkeit zu ihrer Neutralisation mindestens $5-6 \mathrm{ccm} \mathrm{n/10-}$ $\mathrm{NaOH}$. Diese Angabe bezieht sich auf die Blutkörperchen aus ca. $40 \mathrm{ccm}$ Blut, das ist eine Menge, die gerade einen dünnen Belag am Boden des Zentrifugenglases bildet.

Über die Natur der Säure vermag ich augenblicklich noch keine Angaben zu machen. Sie scheint (wenigstens zum Teil) mit Wasserdämpfen überzugehen und dürfte organischer Natur sein.

Während nun das Plasma keine augenscheinlichen Veränderungen zeigt, färben sich die Blutkörperchen im Laufe der Zeit gelblichgrün bis blau. Sie enthalten also eine leicht veränderliche Substanz, die im folgenden als Chromogen bezeichnet werden möge.

\section{Das Chromogen der Blutkörperchen.}

Ich habe zunächst folgenden Weg zur Isolierung dieses Chromogens eingeschlagen. Verrührt man die abzentrifugierten Blutkörperchen mit destilliertem Wasser, so tritt das Chromogen aus und geht in Lösung. Im ersten Augenblick offenbar nur schwach gefärbt, geht der Farbton dieser Lösung schon in wenigen Sekunden je nach der Konzentration in braun bis braunschwarz über. Ich konnte nicht beobachten, daß diese 
Färbung in einer Wasserstoff- oder Kohlensäureatmosphäre verhindert wurde. Es handelt sich also nicht um einen sehr schnell verlaufenden Oxydationsvorgang.

Die Lösung wird zentrifugiert und durch Glaswolle filtriert. Sie ist schwach opalescent und reagiert stark sauer. ${ }^{1}$ ) Diese das native Chromogen enthaltende Lösung bleibt lange Zeit klar, ohne einen Farbenumschlag zu zeigen. Erst nach sehr langem Stehen, z. B. über Nacht, tritt Trübung und Abscheidung blauer Flocken ein.

Durch Aceton wird daraus ein zunächst mißfarbener, in kürzester Zeit aber in tiefblau übergehender Farbstoff gefällt. Alkohol allein wirkt nicht. Es erfolgt nur eine gelbbräunliche Trübung, wohl aber schlägt nachheriger Zusatz von Äther das tiefblaue Chromogen nieder.

Kohlensäure oder Sauerstoff verursachen keine Veränderung in der braunen Lösung.

Durch Mittel, die offenbar nur den Säuregrad der Lösung herabsetzen, werden mißfarbene später in blaugrün und schließlich in blau übergehende Fällungen erzeugt. So genügt schon der Zusatz von einigen Tropfen Leitungswasser, um teilweise Fällungen eintreten zu lassen, während Verdünnung mit destilliertem Wasser nichts ändert. Eine Spur Natriumbicarbonat oder Natriumacetat wirken ebenso. Ebenso fällt bei vorsichtigem Zusatz von n/10-NaOH ein gelbgrünlicher Niederschlag aus, der im Überschuß in Lösung geht. Vorsichtige Zugabe von Säure bringt den Niederschlag wieder zurück, noch weiterer Zusatz löst ihn wieder auf. Sättigung mit $\mathrm{NaCl}$ oder $\mathrm{MgSO}_{4}$ erzeugt gelbbräunliche flockige Ausfällung, desgleichen Ferrocyankalium. Pepton fällt sofort. Der Niederschlag wird schnell blau. Alle diese letztgenannten Mittel fällen, wie ich vermute, das Chromogen nur in unvollkommen reduziertem Zustande.

Anders, wenn gewisse organische reduzierende (?) Substanzen in Anwendung kommen.

So erhält man prachtvolle, tief blauschwarze Ausfällungen durch Pyrrogallol, Brenzkatechin oder auch Tannin. Selbst der

1) Zurück bleiben die Stromata und wohl auch etwas von dem vielleicht durch Reduktionswirkung gefällten Farbstoff, cf. unten. 
spurweise Gehalt einer Lösung an Chromogen läßt sich mit genannten Substanzen noch deutlich nachweisen. Tannin und Pyrogallol scheinen schon in saurer Lösung zu wirken, während bei Anwendung von Pyrogallol noch der Zusatz einer säureabstumpfenden Substanz nötig ist; die Farbtöne sind aber dann um so schöner. Auch mit dem frischen Blut treten diese Reaktionen ein und selbst im mikroskopischen Präparat lassen sich die Blutkörperchen durch Pyrogallol färben.

\section{Der Vanadiumgehalt des Chromogens.}

Bisher wurde das blaue Chromogen stets durch Ausfällung mittels Aceton aus der braunen wässerigen Lösung des nativen Chromogens, wie man sie durch Hydrolyse der Blutkörperchen mit destilliertem Wasser erhält, dargestellt. Aceton schien mir das einfachste und wenigst eingreifende Mittel zu sein. Der Farbstoff wurde zentrifugiert, mehrfach gut mit verdünntem Alkohol gewaschen und auf einem gewogenen Filter gesammelt.

Beim Veraschen dieses Chromogens und Abrauchen mit Salpetersäure hinterbleibt nach dem Glühen ein rotorange gefärbter, geschmolzener Rückstand, der sich als Vanadinsäure $\left(\mathrm{Vd}_{2} \mathrm{O}_{6}\right)$ erwies.

Diesen überraschenden Befund habe ich auf folgendem Wege sicherzustellen gesucht:

Der Glührückstand löst sich in $\mathrm{HCl}$ oder $\mathrm{HNO}_{3}$ mit gelber, eventuell ins Grüne übergehender Farbe.

Die Reaktionen auf Fe (Rhodan- und Ferrocycankaliumreaktion), auf $\mathrm{Mn}$ (Oxydationsschmelze), Ni (Diacetyldioximreaktion) waren absolut negativ. Diese Metalle kamen infolge der Färbung der zu prüfenden Lösung zuerst in Frage.

Bei der Prüfung nach dem gewöhnlichen analytischen Gang ergab sich folgendes: $\mathrm{H}_{2} \mathrm{~S}$ in Lösung: Keine Fällung, nur Übergang der Farbe in grünlichblau. $\mathrm{NH}_{3}$ bei Gegenwart von $\left(\mathrm{NH}_{4}\right) \mathrm{Cl}$ : nichts. $\left(\mathrm{NH}_{4}\right)_{2} \mathrm{~S}$ : Braunfärbung der Lösung, keine Fällung.

Da also die Reaktionen auf die bekannten Metalle sämtlich negativ ausfielen, konnte es sich nur noch um ein seltenes Element handeln. Vanadium kam infolge des eigentümlichen 
Farbenwechsels der Lösungen in erster Linie in Betracht, und alle speziellen Reaktionen haben dies bestätigt.

Der mit $\mathrm{HNO}_{3}$ abgerauchte Glührückstand ist tief orangebraun, glühbeständig, schmelzbar $\left(\mathrm{Vd}_{2} \mathrm{O}_{\overline{5}}\right)$. Er löst sich in Säuren zu stark gefärbten Salzen, aber auch in Alkalien und Ammoniak zu vanadsauren Salzen, die farblos sind. Säuren färben die Lösungen der letzteren gelb und grüngelb.

Durch Zink werden die angesäuerten Lösungen reduziert, wobei die anfangs gelbe Farbe der Lösung in grün und blau (Dioxyd), später in blaugrün (Sesquioxyd) und schließlich in rein blau, sogenanntes lavendelblau (Oxydul) übergeht.

Die sauren Lösungen werden beim Kochen mit Oxalsäure oder Weinsäure usw. reduziert und blau. Wasserstoffsuperoxyd färbt saure Lösungen tief rot. Die Farbe geht nicht in Äther über.

Eine andere sehr empfindliche und von Ellram ${ }^{1}$ ) angegebene Farbenreaktion tritt prompt ein. Gibt man nämlich Rhodankalium zu einer selbst Spuren enthaltenden Lösung eines vanadsauren Salzes, so erhält man bei vorsichtigem, tropfenweisem Zusatz von konzentrierter Schwefelsäure eine tief blaue Lösung.

Silbernitrat und Bleiacetat fällen gelbe Niederschläge.

Besonders charakteristisch verhielt sich ferner eine kleine Menge der aus dem Chromogen erhaltenen Vanadsäure. Es gelang damit, eine große Quantität salzsauren Anilins, dem etwas chlorsaures Kalium zugefügt wurde, zu Anilinschwarz zu oxydieren. Die in einem kleinen Becherglase angesetzte Lösung erstarrte in kurzer Zeit zu einem dicken Brei von Anilinschwarz.

Auch mit Tannin gibt die mit Essigsäure angesäuerte Lösung eine schwarzblaue Fällung (Berzelius). Prachtvolle tiefblaue Färbungen, resp. Fällungen, erhält man ferner mit Pyrogallol und auch mit Brenzkatechin. In der Literatur fand sich darüber keine Angabe. Es ist dies jedoch bemerkenswert, da, wie oben gezeigt wurde, dieselben Fällungen auch mit dem nativen Chromogen eintreten.

1) W. Ellram, Über einige neue Reaktionen von vanadinsauren Molybdänsäuren und Rhodanverbindungen, Sitzungsberichte der Dorpater Naturforschenden Gesellschaft, 1895, S. 281. 
Alle diese Reaktionen lassen keinen Zweifel mehr, daß es sich tatsächlich um Vanadium handelt. Das Chromogen in den Blutkörperchen der Ascidien ist also eine Vanadiumverbindung. Sehr wahrscheinlich tritt das seltene Element darin in Form der Vanadinsäure auf, denn die Reaktionen mit Tannin, Pyrogallol und Brenzkatechin treten mit dem Chromogen genau so ein, wie mit der Vanadinsäure selbst. Weitere Spekulationen ohne experimentelle Unterlagen sind verfrüht, es ist aber infolge der Analogie der Vanadinsäure mit der Phosphorsäure verführerisch, an Verbindungen von der Konstitution der Nucleinsäuren zu denken. Ich hoffe durch weitere Untersuchungen hier Aufschluß geben zu können.

Frägt man sich nach der Bedeutung solcher Vanadverbindungen in der Zelle, so liegt nichts näher, als. sich der stark katalytischen, speziell Sauerstoff übertragenden Wirkung der Vanadinsäure zu erinnern. Im chemischen Laboratorium macht man ausgedehnten Gebrauch davon. Ich erinnere nur an die Entdeckung von Witz: ${ }^{1}$ ) Ein Teil Vanadinsäure genügt, um 270000 Teile Anilin (bei $\mathrm{KClO}_{3}$-Zusatz) in Anilinschwarz überzuführen.

$\mathrm{Daß}$ auch im chemischen Laboratorium der Zelle dieser Verbindung analoge Aufgaben zufallen, d. h. Oxydationen zu vermitteln, dürfte keine zu gewagte Vermutung sein.

Eine, wenn auch mit wenig Material ausgeführte quantitative Bestimmung des Vanadiumgehaltes des Chromogens ergab einen Gehalt von über $15 \% \mathrm{Vd}_{2} \mathrm{O}_{5}$.

Zum Schluß sei erwähnt, daß es bisher auch bei einer anderen Ascidienart, nämlich Ascidia mentula, gelang, Vanadium nachzuweisen.

1) G. Witz, Über die industrielle Anwendung der Vanadinsäure in der Anilinschwarzfabrikation, C. R. A. S., Bd. 83, S. 348. 